\title{
MUJERES CON DISCAPACIDAD: UN RETO PARA LA TEORÍA FEMINISTA
}

\author{
LAURA VIÑUELA SUÁREZ \\ Espora Consultoría de Género (Gijón)
}

Recibido: 06/04/2009

Aceptado: 28/05/2009

«La discapacidad, como el género y la raza, está en todas partes, una vez que sabemos cómo encontrarla»

Rosemarie Garland-Thomson ${ }^{1}$

Los estudios feministas de la discapacidad se han desarrollado muy recientemente. Igual que sucedió en otros campos del saber, este tipo de análisis se inició en el mundo anglosajón y, de forma más destacada, en el campo de las Ciencias Sociales. Tanto los estudios de la discapacidad como los estudios feministas vienen impulsados, en el ámbito académico, por un movimiento social previo. Así, del mismo modo que la lucha de las mujeres en pro de su igualdad de derechos comenzó mucho antes de que se empezara a analizar su situación y el sistema dominante que la sustentaba, también el campo de los estudios de la discapacidad nace de las reivindicaciones del movimiento de personas con discapacidad. El discurso hegemónico impregna todos los campos del saber y han sido las personas que sufren la opresión del sistema de valores dominante las que lo han cuestionado y han promovido su desconstrucción.

Durante largo tiempo, los estudios sobre discapacidad estuvieron confinados en el ámbito de la medicina, en tanto en cuanto «discapacidad» se relacionaba exclusivamente con «enfermedad». En este marco se desarrolló lo

1. GARLAND-ThOMSON, Rosemarie. Re-shaping, Re-thinking, Re-defining: Feminist Disability Studies. Washington D.C., Center for Women Policy Studies, 2001, p. 20.

* Todas las citas han sido traducidas del inglés por la autora de este artículo.

Feminismo/s 13, junio 2009, pp. 33-48 
que se conoce como el «modelo médico de la discapacidad», que la considera como algo únicamente biológico y patológico, centrándose en la necesidad de «curarla». Sólo cuando el movimiento de personas con discapacidad comenzó a ser más fuerte se puso en cuestión esta interpretación y se enfatizó la importancia de la configuración social en el grado de discapacitación de las personas, naciendo así el «modelo social de la discapacidad». Paralelamente a este nuevo enfoque, se desarrollaron propiamente los llamados «estudios de la discapacidad» («Disability Studies», en inglés).

Las décadas de 1970 y 1980 fueron fundamentales para la consolidación tanto de los estudios feministas como de los estudios de la discapacidad en el ámbito académico. Sin embargo, la unión de estos dos campos en lo que la estudiosa norteamericana Rosemarie Garland-Thomson llama «estudios feministas de la discapacidad» aún debió esperar un tiempo. En la última década del siglo pasado estos dos ámbitos empezaron a confluir ya que hasta ese momento, si bien existían algunas publicaciones, ni los estudios feministas habían centrado su atención en la discapacidad, ni los estudios de la discapacidad habían tenido en cuenta de forma sistemática las necesidades específicas de las mujeres.

En España estos estudios son aún prácticamente inexistentes. En primer lugar, es necesario tener en cuenta la trayectoria histórica de nuestro país, que sólo en 1975 salió de una larga dictadura, lo que implica que la apertura del ámbito académico a estudios no tradicionales es relativamente reciente. La evolución de los estudios de las mujeres en España muestra la estrecha relación entre el movimiento feminista y el desarrollo de los estudios de género en la universidad española y pone de manifiesto el notable avance conseguido en muy pocas décadas.

Del mismo modo, es posible trazar una trayectoria similar para los estudios de la discapacidad. El movimiento de personas con discapacidad se inició tímidamente con la relativa apertura que caracterizó los últimos años del régimen franquista y se consolidó a partir de finales de la década de los 70, con la aparición de los primeros grupos de marcado carácter reivindicativo. El movimiento asociativo de mujeres con discapacidad en España es aún más reciente y no es hasta los años 90 cuando aparecen las primeras asociaciones (Dones No Estàndards, de Barcelona, se crea en 1995). El desarrollo más fuerte del movimiento organizado de mujeres con discapacidad se ha dado ya en el presente siglo, con el nacimiento de nuevas asociaciones (AMDAS La Fonte, en Asturias, o Asociación Luna, en Almería, ambas en 2001) o la creación en el 
año 2000 de la Comisión de la Mujer del Comité Español de Representantes de Personas con Discapacidad - CERMI².

Si tenemos en cuenta el impulso que tanto los estudios feministas como los estudios de la discapacidad han recibido desde los movimientos asociativos, no es de extrañar el escaso desarrollo de los estudios feministas de la discapacidad en España. La mayor parte de los estudios sobre mujer y discapacidad en nuestro país se enmarcan en las Ciencias Sociales y de la Salud y, en general, se centran en análisis de la situación de las mujeres con discapacidades. Sus conclusiones se orientan a menudo al diseño de medidas específicas para corregir las desigualdades existentes en diversas áreas a través del desarrollo de políticas, planes o protocolos y métodos de intervención.

Este tipo de investigaciones es fundamental y ha supuesto una mejora de las condiciones de vida de las mujeres con discapacidad, a la vez que pone de relieve la importancia de considerar sus necesidades y situaciones de forma específica. Sin embargo, resulta clave el desarrollo paralelo de los estudios feministas de la discapacidad desde las humanidades. Este enfoque promueve la realización de análisis teóricos y filosóficos sobre las categorías «mujer» y «discapacidad», así como de las relaciones entre ambas, a fin de sacar a la luz las ideologías subyacentes en estas nociones y comprender cómo articulan tanto las vidas cotidianas de las mujeres con discapacidad como el modo en que la sociedad responde (o no) a sus necesidades e intereses. Este punto de vista es el que se tratará en el presente artículo y resulta novedoso en el propio ámbito anglosajón, donde es defendido por autoras como Barbara Hillyer ${ }^{3}$ o Rosemarie Garland-Thomson: «Es necesario estudiar la discapacidad en el contexto de lo que consideramos el corpus de conocimiento que cuenta la historia de nuestro mundo y nuestras vidas - las Humanidades» ${ }^{4}$.

Como afirma Anita Silvers ${ }^{5}$, la teoría feminista, aunque aún no ha incorporado de forma generalizada la perspectiva de la discapacidad, ha sido con diferencia uno de los ámbitos filosóficos que mayor atención le ha prestado desde el punto de vista de la identidad. Sin duda, la propia conceptualización

2. Ríu, Carmen y Laura Viñuela. Módulo específico de formación en género y discapacidad. Oviedo, Fundación FASAD, 2004, pp. 75-82.

3. HillyeR, Barbara. Feminism and disability. Norman and London, University of Oklahoma Press, 1993.

4. Garland-Thomson, Rosemarie. Re-shaping, Re-thinking... Op. cit., p. 2.

5. SiLverS, Anita. «Feminist Perspectives on Disability». Stanford Encyclopedia of Philosophy, 2009.

Feminismo/s 13, junio 2009, pp. 33-48 
histórica de las mujeres como discapacitadas ${ }^{6}$ ha sido de vital importancia para acercar ambos campos.

Uno de los puntos de encuentro más claros de la teoría feminista y la discapacidad es el relativo al cuerpo y sus conceptualizaciones. A través de la sujeción de la identidad de las mujeres a sus cuerpos (maternidad y sexualidad), el discurso patriarcal ha justificado su inferioridad e, incluso, su no humanidad; en consecuencia, ha intervenido sobre los cuerpos femeninos para eliminar o disciplinar, en términos foucaldianos, sus «anormalidades» (cliterectomías o histerectomías son los ejemplos más evidentes).

En relación a esto, para apoyar filosóficamente la inferioridad de las mujeres y de las personas con discapacidad, ha sido fundamental la idea cartesiana que establece una separación entre cuerpo y mente, considerando a la segunda superior y más importante, de modo que se desea trascender el cuerpo y alejar lo más posible al sujeto «puro» (mental) de su corporeidad. Esta dualidad es clave para el sistema patriarcal, que privilegia las actividades relacionadas con la mente sobre todo lo que tiene que ver con el cuerpo. Así, reduce tanto a mujeres como a personas con discapacidad a pura corporeidad al ser sus (dis)funciones corporales más evidentes - menstruación, maternidad, discapacidades físicas visibles y, más aún, discapacidades intelectuales y mentales-, relegándolas a un plano inferior y enfatizando lo corporal hasta el punto de limitar sus identidades exclusivamente a ese aspecto, negando e invisibilizando otros elementos identitarios.

Para el ideal que iguala el sujeto a la mente, cualquier persona cuya corporeidad esté en un primer plano es amenazadora, porque pone en evidencia la falacia del propio sistema y demuestra que no es posible pensar en un sujeto puramente mental, unitario e independiente de las constricciones de lo físico. Para mantener la idea de que el sujeto es independiente de su corporeidad, los cuerpos «desviados», es decir, los que rebaten esta idea (deformes, cambiantes, problemáticos, necesitados de cuidado) han sido controlados y disciplinados de diversas formas por el sistema dominante, patriarcal y capaz. El control histórico sobre sus cuerpos es una característica común de mujeres y personas con discapacidad.

Una teórica clave en esta cuestión es Julia Kristeva ${ }^{7}$. Esta autora parte de las afirmaciones de Mary Douglas (1966, Purity and Danger) que explica có-

6. SHERWIN, Susan. No Longer Patient: Feminist Ethics and Health Care. Philadelphia, PA, Temple University Press, 1992.

7. Moreno Álvarez, Alejandra. Lenguajes comestibles: Anorexia, bulimia y su descodificación en la ficción de Margaret Atwood y Fay Weldon. Palma, Universitat de les Illes Balears, Treballs Feministes, 2009. 
mo la estructuración social se basa en la dicotomía que iguala orden a pureza, por oposición a desorden y contaminación, y las elabora explicando cómo el patriarcado ha trasladado estos dualismos al cuerpo de las mujeres para legitimar el control sobre ellas. Así, según Kristeva, existe una correspondencia entre la integridad del cuerpo y la integridad del sujeto. Sin embargo, el cuerpo femenino es cambiante -maternidad- y sus fronteras poco claras -menstruación, fluidos-, algo que también sucede con el cuerpo discapacitado, cuyas «faltas» son, en general, evidentes. Esto es de gran relevancia para explicar por qué las discapacidades sólo son consideradas socialmente como tal si son visibles, por qué se han realizado esfuerzos médicos por «normalizar», es decir, invisibilizar, ciertas discapacidades o por qué algunas personas con discapacidad tratan de esconderlas, a fin de evitar el rechazo social que provoca tener una discapacidad.

Al poner en cuestión la separación cuerpo-mente, el feminismo y los estudios de la discapacidad afirman que todos los sujetos, también aquéllos a los que se refiere el dualismo cartesiano y patriarcal, están unidos inevitablemente a sus cuerpos, con lo cual se abre el camino para una nueva concepción de lo que significa ser sujeto e individuo. Sin embargo, el énfasis en la mente como el lugar de existencia del sujeto ha sido también muy relevante para las reivindicaciones de igualdad de las mujeres y de las personas con discapacidad: una vez que se admite que ser mujer o tener una discapacidad física o sensorial no implica una inferioridad de la mente, se admite también que es posible ser sujeto en un cuerpo distinto al normativo (masculino y capaz). De este modo, estratégicamente, el dualismo cuerpo-mente ha sido liberador para quienes a lo largo de la historia han sido reducidos únicamente a cuerpo y ha resultado útil para el reconocimiento de que las personas con cuerpos «diferentes» (femeninos y/o discapacitados) son sujetos y tienen capacidad de decisión.

Esta idea de sujeto centrada en las capacidades de la mente aún persiste en las argumentaciones para reivindicar el derecho a la igualdad de los grupos de personas caracterizados y subordinados por el discurso hegemónico en función de sus diferencias físicas (se dice, por ejemplo, que las mujeres debemos tener las mismas oportunidades que los hombres porque intelectual y mentalmente tenemos las mismas capacidades que ellos, y lo mismo sucede para otros grupos de personas en situaciones de discriminación). Sin embargo, pese a su valor estratégico en determinados casos, aplicar una perspectiva de discapacidad exige una revisión de este argumento, ya que no puede utilizarse sin más a la hora de reivindicar los derechos de las personas cuyas capacidades mentales e intelectuales se alejan de lo que se considera «normal». 
Es un clásico en la historia del feminismo el peligro que suponen las generalizaciones, corriendo así el riesgo de caer en la práctica patriarcal de hablar por las demás. Desde el punto de vista de las mujeres con discapacidad, un tema a tener en cuenta es qué hacer cuando se trata de mujeres que no pueden hablar por sí mismas debido a la gravedad de su discapacidad. Desde sus inicios, diversos grupos de mujeres han ido reclamando al movimiento feminista y a su teoría un espacio propio para hablar por sí mismas de sus necesidades y su situación. El ejemplo más conocido es el cuestionamiento de las feministas negras de los presupuestos feministas hasta los 70, cuando el movimiento había sido dirigido por mujeres blancas y de clase media-alta.

Una característica fundamental del feminismo es que, desde el primer momento, fue capaz de revisar sus planteamientos para acomodar una multiplicidad de voces. Algo que ha continuado haciendo constantemente, sin perder, al mismo tiempo, la capacidad para funcionar como un movimiento político organizado (ha sabido combinar la multiplicidad de las mujeres con la unidad en sus reivindicaciones). Por esa razón se habla actualmente de «feminismos» más que de «feminismo». Evidentemente, aún existen muchas voces que deben dejarse oír dentro de estos feminismos, y la de las mujeres con discapacidad es una de ellas. Barbara Hillyer aborda la cuestión de la autonomía y la capacidad de expresarse una misma en su libro Disability and feminism, cuando sus ideas como feminista chocan con la situación de su hija con una discapacidad severa. «Hemos llegado a comprender que la capacidad de una mujer para valerse por sí misma es crucial para el mantenimiento de sus opciones, pero algunas personas discapacitadas no son capaces de valerse por sí mismas» ${ }^{8}$.

Otro punto en el que feminismo y discapacidad convergen es en el establecimiento de una diferencia entre lo biológico y lo social. El feminismo encontró una gran fuerza en la posibilidad de separar las características biológicas del cuerpo femenino de los roles que se le atribuyen. Así, la distinción entre «sexo», referido a las características físicas que identifican a un cuerpo humano como de mujer, $\mathrm{y}$ "género», referido a las características sociales que se adscriben a un cuerpo sexuado como femenino, ofreció al feminismo un escape a las teorías esencialistas que fijaban la identidad femenina en parámetros de debilidad, cuidado, sacrificio e inferioridad porque se consideraba que estas características estaban predeterminadas biológicamente (y, por tanto, eran inmutables). Una vez que se puso de manifiesto el carácter construido de los atributos «femeninos», las feministas pudieron comenzar a reivindicar

8. Hillyer, Barbara. Feminism and disability... Op. cit., p. 7.

Feminismo/s 13, junio 2009, pp. 33-48 
opciones diferentes para las mujeres y se abrió un nuevo camino en que las mujeres podían elegir con mayor libertad.

Del mismo modo, para las personas con discapacidad ha sido fundamental poder separar la «disfunción» (impairment en inglés) de la «discapacidad» ${ }^{9}$. Jenny Morris cita las definiciones que hace de tales términos el British Council of Disabled People: «disfunción» es la característica del cuerpo de una persona que afecta a su apariencia en un modo no aceptado socialmente, al funcionamiento de su mente o su cuerpo, y que puede causar dolor, fatiga, afectar la comunicación o reducir la consciencia, y «discapacidad» se entiende como la restricción en la actividad personal causada por un sistema social que no tiene en cuenta las necesidades de las personas con «disfunciones» ${ }^{10}$. Así, «las personas discapacitadas son aquéllas con disfunciones que son discapacitadas por la sociedad ${ }^{11}$.

Esta separación marca el cambio entre el «modelo médico-biológico»y el «modelo social»de la discapacidad. El primero que considera a la persona con discapacidad como una enferma que sufre un problema y a la discapacidad como algo que hay que curar y que entiende la discapacidad como una desgracia personal y un problema individual. A su vez, este segundo«modelo social» de la discapacidad, cambia el enfoque hacia los factores sociales que hacen que una persona con determinadas diferencias biológicas no pueda realizar sin esfuerzo algunas actividades. Este modelo pone de relieve las asunciones ideológicas de «normalidad»y «capacidad» que subyacen en nuestro modelo social y es liberador en tanto en cuanto dota a las personas con discapacidad

9. La cuestión de la terminología para referirse a las personas con discapacidad es interesante y existen diferentes opciones. En este caso, se utiliza "personas discapacitadas» siguiendo las preferencias británicas que ponen así énfasis en el papel que la sociedad tiene como factor discapacitador. En el ámbito de los Estados Unidos en más común el uso de "personas con discapacidades», con el objetivo de poner de relieve que la discapacidad es sólo una más de las muchas características que conforman a una persona, y no la única. En nuestro país, Soledad Arnau Ripollés prefiere hablar de "personas con diversidad funcional», nomenclatura acuñada por el Foro de Vida Independiente, a fin de evitar el carácter peyorativo que se asocia al término «discapacidad» y poner de manifiesto que las diferencias en el funcionamiento de determinadas personas no llevan aparejada una menor valía. Asimismo, desde la asociación Dones No Estàndards, se habla de "personas no estándar», enfatizando el hecho de que todas las personas tienen algún aspecto que no se ajusta a la normalidad y que esto favorece la riqueza social y cultural.

10. MORRIS, Jenny. «Feminism, gender and disability». Ponencia presentada en un seminario en Sidney, Australia, 1998, p. 2.

11. British Council of Disabled People, citado en MORRIS, Jenny. «Feminism, gender and disability»... Op. cit., p. 3. 
de las herramientas para describir sus experiencias de discriminación más allá de una incapacidad individual y determinada biológicamente ${ }^{12}$.

En ambos casos, este paso desde el determinismo biológico hasta las constricciones social y culturalmente impuestas pasa por el conocido eslogan «lo personal es político» que articuló el movimiento feminista en la década de los '70. El reconocimiento de que los problemas que una persona encuentra en su vida cotidiana no son únicos e individuales, sino compartidos por otras muchas, es fundamental para la toma de conciencia comunitaria que precede al desarrollo de una acción política colectiva encaminada a la solución de esos problemas.

A la vez que es posible identificar, como hemos visto, numerosos puntos de encuentro entre los estudios feministas y los estudios de la discapacidad, existen también otros aspectos que presentan mayores complicaciones $y$, por tanto, interesantes retos. Por ejemplo, mientras que para el feminismo los roles de género tradicionales constituyen claramente un elemento opresor para las mujeres, cuando pensamos en ellos desde el punto de vista de las mujeres con discapacidad esta afirmación se complica. Como hemos visto más arriba, las mujeres con discapacidad no se han considerado tradicionalmente como «mujeres», sino que han estado confinadas a una categoría distinta, no sexuada, y no se les ha permitido el acceso a los elementos que construyen la categoría patriarcal «mujer» (belleza, sexualidad, maternidad, cuidado), por más represores que éstos hayan podido ser.

Desde este punto de vista, para las mujeres con discapacidad puede ser positivo el acceder a ciertos elementos estereotipados del rol de género femenino, ya que así pueden reivindicar su derecho a ser consideradas mujeres y a desarrollar una identidad de género que históricamente se les ha negado. Para poder rechazar los estereotipos relacionados con las mujeres es necesario, en primer lugar, que se considere que esos estereotipos les son aplicables. Por esta razón, reclamar su derecho a la belleza, a la sexualidad, a la maternidad (elementos que continúan siendo en gran medida opresores para las mujeres sin discapacidad) puede ser empoderante para las mujeres con discapacidad.

Sin embargo, esto presenta dificultades para las feministas, que llaman la atención sobre los peligros potenciales de esta estrategia: «una capacidad de acción que evita el estereotipo en función de la discapacidad puede llevar a un estereotipo en función del rol sexual $»^{13}$. Además, la experiencia del movimiento feminista prueba que el deseo de «normalización» (en este caso, de

12. MORRIS, Jenny. «Feminism, gender and disability»... Op. cit., p. 3.

13. Hillyer, Barbara. Feminism and disability... Op. cit., p. 7. 
acceder al status de lo que se considera una mujer «normal» en el sentido tradicional) a menudo resulta en una aceptación de la norma patriarcal, con todas las consecuencias negativas que esto conlleva, e implica también la invisibilización de lo que es diferente a la norma. Así, es fundamental que el proceso a través del cual las mujeres con discapacidad adquieran identidad como «mujeres» ofrezca más posibilidades que a través de las características patriarcales adscritas a tal categoría, a fin de evitar sus efectos negativos. En este punto es muy significativo el desarrollo de los estudios feministas de la discapacidad, que pueden ofrecer una línea de pensamiento alternativa y muy enriquecedora.

Por un lado, el feminismo tiene experiencia en cuanto a las posibilidades de reapropiarse de elementos tradicionalmente opresores para darles otro significado. Por ejemplo, en los '70 las feministas criticaron el estándar de belleza femenina y eligieron apartarse de él, rechazando todos los elementos que reforzaran el estereotipo de la feminidad (tacones, melenas, maquillaje, etc.). Así, se estableció un canon estético que el patriarcado se encargó de devolverles con una connotación negativa creando el estereotipo de las feministas como mujeres feas y descuidadas. De este modo, se difundió un modelo que la mayoría de las mujeres no querrían emular, lo que neutralizó en gran medida la capacidad feminista para promover el cambio a través de la acción colectiva.

En consecuencia, a través de un estereotipo basado en la apariencia quedó impedido el poder transformador del feminismo de los ' 70 y se inició un largo proceso de lucha contra este modelo peyorativo que abrió debates dentro del propio movimiento que aún continúan vivos y que son una muestra más de la maleabilidad del feminismo para acomodar en su seno visiones muy diferentes. Resulta interesante para el tema que nos ocupa que, gracias precisamente a que en los '70 las feministas expresaron su rechazo al modelo de belleza femenina tradicional, las feministas actuales pueden ahora reapropiarse de elementos de bricolaje estético «femenino», dotarlos de nuevo significado y utilizarlos sin que eso implique una aceptación del modelo patriarcal de belleza. En este sentido se expresa, por ejemplo, Janet Radcliffe Richards en The Sceptical Feminist (1980), que revaloriza el poder del artificio como forma de resistencia y «ve la polisemia del pluralismo y el juego del artificio en las formas de vestir de la cultura postmoderna como una estrategia más apropiada para mostrar políticas corporales individuales de consenso o de disidencia» ${ }^{14}$.

14. En CARSON, Fiona. «Feminism and the Body». London y New York, Routledge, 2001, p. 126.

Feminismo/s 13, junio 2009, pp. 33-48 
Esto es interesante desde el punto de vista de las mujeres con discapacidad, en el sentido que mencionábamos antes de reclamar para sí elementos identitarios femeninos como estrategia empoderante, a pesar de que tales elementos hayan sido considerados opresores desde el punto de vista feminista. Recordemos que discapacidad y belleza se consideran términos excluyentes (en todo caso, se puede ser «bella a pesar de...») y que, dada la importancia de la belleza en la identidad femenina tradicional, esta dicotomía abunda en la exclusión de las mujeres con discapacidad de la categoría «mujer». Desde esta perspectiva, reclamar la belleza puede ser una estrategia para poner en cuestión un estereotipo.

Hay que tener en cuenta también que esto no tiene por qué significar una vuelta a la categoría de belleza como algo opresivo y que no necesariamente se reclama un estándar de belleza femenina tradicional (algo que, por otra parte, y como todos los modelos femeninos idealizados, es imposible de alcanzar). Para lograr esto, entonces, es necesario problematizar la idea de belleza, reclamarla para dotarla de un significado diferente. Por otro lado, reclamar el acceso a la feminidad tradicional tampoco tiene por qué ser la estrategia más adecuada, sino que puede reclamarse el derecho a que un cuerpo diferente sea aceptado y visto como bello y aprovechar los elementos de resistencia que hay en la diferencia.

En este sentido, es posible encontrar un punto de vista interesante en los estudios sobre lo grotesco y lo carnavalesco de Bakhtin y, más directamente aplicado al cuerpo de las mujeres, de Mary Russo, quien dice:

el cuerpo clásico es trascendente, monumental, cerrado, estático, autocontenido, simétrico y pulcro; se identifica con la «alta» cultura o cultura oficial del Renacimiento y, más tarde, con las aspiraciones racionalistas, individualistas y normalizadoras de la burguesía. El cuerpo grotesco es abierto, protuberante, irregular, que excreta, múltiple y cambiante; se identifica con la cultura no oficial, «baja» o carnavalesca y con la transformación social ${ }^{15}$.

La idea de resistencia a través del reclamo de lo que se considera grotesco es una propuesta útil y, en el campo de las mujeres con discapacidad, permite ir más allá de la superación heroica de la discapacidad o la victimización: es una forma diferente de poner en primer plano y visibilizar las implicaciones de la discapacidad, sin negarla pero, al mismo tiempo, sin victimizarla. Sería una reapropiación del cuerpo discapacitado, una alternativa empoderadora a la corrección política. Las artistas feministas, con y sin discapacidad, han trabajado con éxito en este ámbito en las últimas décadas para cuestionar

15. Russo, Mary. The Female Grotesque: Risk, Excess, and Modernity. New York, Routledge, 1994. 
elementos de la lógica patriarcal desde el punto de vista de sus consecuencias sobre las vidas de las mujeres. Un ejemplo que contó con gran repercusión mediática fue la monumental escultura «Alison Lapper Embarazada» que se expuso en Trafalgar Square (Londres) en 2005, mostrando a Alison Lapper, que nació sin brazos ni manos y con las piernas muy cortas debido a la focomelia, en un estado muy avanzado de embarazo. Esta obra utiliza el arte para visibilizar un cuerpo en el que coexisten nociones incompatibles en el imaginario patriarcal: sexualidad, maternidad y discapacidad, obligando así a quienes miran a enfrentarse a estas diferencias y poniendo en cuestión mitos fundamentales del sistema dominante.

Si bien la idea de belleza no se ha asociado a las mujeres con discapacidad, hay otra igualmente opresora que sí se ha relacionado con ellas: la de las heroínas, referida a aquéllas que han logrado «superar» su discapacidad, restándole importancia, negando los aspectos de sufrimiento y dolor. Jenny Morris afirma que esta creación de relatos heroicos que tratan de esconder las dificultades se deben al miedo a la victimización, pero esto resulta problemático porque se propone un modelo de superwoman. Este es otro de los puntos en los que el feminismo tiene también experiencia, porque la mujer que puede con todo (cuidado, trabajo, vida social, belleza) es una imagen que se iguala al éxito y se presenta como lo contrario de la mujer débil y víctima. Sin embargo, este modelo es peligroso porque puede producir mucho estrés por la sobreexigencia y la necesaria ocultación de las dificultades.

Un modelo social que pone énfasis en la productividad y la independencia y la autonomía (el no necesitar a otros, el no ser una carga para otros, y esto último es relevante para el feminismo porque esos otros para quienes se es carga a menudo son mujeres, como comentamos en el apartado anterior) favorece este tipo de historias. Hillyer habla de las implicaciones negativas que tienen el descanso o la lentitud en una cultura que valora a las personas en función de su productividad (algo que, por otro lado, estaba detrás de muchas acciones históricas de eliminación de personas con discapacidad: no valen para nada, no producen nada, sólo consumen, no pueden mantenerse por sí mismas). Según Hillyer, «bajar el ritmo en una cultura que se mueve rápido significa apartarse de la norma» ${ }^{16}$.

En consecuencia, la norma es lo deseable y, en los relatos de heroicidad, se idealiza a las personas que, siendo distintas al modelo predominante, se esfuerzan al máximo por ajustarse a él (produciendo lo más posible, tratando de acomodar su apariencia a los cánones, ocultando sus diferencias y negando

16. HILLYER, Barbara. Feminism and disability... Op. cit., p. 54.

Feminismo/s 13, junio 2009, pp. 33-48 
sus dificultades). De este modo, se refuerza como deseable el modelo dominante y se evita que las personas «normales» tengan que confrontar la existencia de la desviación que, en último término, cuestiona ese modelo. Las consecuencias son negativas en lo personal:

Lentamente empecé a darme cuenta de cómo, durante toda mi vida, había trabajado muy duro para estar 'bien adaptada' y asegurarme de que así es como me veían los demás. Y empezó a hacerse evidente qué significaba eso. Significa sonreír cuando sentía dolor y tranquilizar a quien estuviera conmigo. Significaba referirme a mi pierna sólo si tenía algo divertido que contar sobre ella. Significaba aceptar cualquier cosa que me hicieran los médicos (psicológica y físicamente) con valor incondicional. En definitiva, significaba ser muy desleal conmigo misma ${ }^{17}$.

Esto no quiere decir que haya que centrarse en las dificultades y la solución tampoco es la aceptación sin más de la discapacidad en el sentido de presentarla como algo deseable. Hillyer cita a Nancy Frick en un congreso sobre el síndrome post-polio: «Por aceptación no quiero decir aprender a preferir la discapacidad sobre la capacidad. Tampoco quiero decir resignarse a lo inevitable. Me refiero a las condiciones que hacen posible ver la propia discapacidad como algo más que un defecto» ${ }^{18}$.

Hillyer llama la atención sobre la importancia de tener todas estas tensiones en cuenta. La cuestión no es elegir entre negar que la discapacidad es difícil o poner de relieve esas dificultades, sino que hay que atender a ambas dimensiones. Por eso es clave la propuesta de Garland-Thomson, que aboga por la conveniencia de la multiplicidad como el camino más adecuado: no hay que negar un binarismo creando otro, no hay que hablar sólo de lo social y esconder lo biológico, no hay que enfatizar la fuerza y esconder la debilidad, sino que hay que cuestionar un modelo que nos obliga a elegir. La propia Hillyer lo expresa con claridad en la siguiente cita:

Las experiencias de la discapacidad que pueden enriquecer el desarrollo de la teoría feminista (...) incluyen el reconocimiento de que (...) la senilidad y las enfermedades degenerativas minan la creencia en la vida como un proceso de crecimiento; que el conocimiento total e interiorizado de la condición 'real' del cuerpo permite la eliminación del heroísmo falso o excesivo; que los seres humanos son limitados; que algunas pérdidas no pueden repararse; y, sobre todo, que es necesario integrar tanto la fuerza como la debilidad femeninas ${ }^{19}$.

17. En Hillyer, Barbara. Feminism and disability... Op. cit., p. 113.

18. En Hillyer, Barbara. Feminism and disability... Op. cit., p. 117.

19. Hillyer, Barbara. Feminism and disability... Op. cit., p. 15. 
Todos estos aspectos teóricos comentados hasta ahora no son simples abstracciones, sino que tienen una influencia directa y bien visible en las vidas cotidianas de las mujeres con discapacidad. Que hayan sido consideradas como «no-humanas» $y$ «no-mujeres» influye en su autopercepción y su autoestima, así como en la percepción que el resto de las personas tienen de ellas como seres necesitados de supervisión constante, incapaces de tomar decisiones por sí mismas y, por esta razón, a menudo infantilizadas. Esta visión merma sus derechos de ciudadanía, algo que muestran claramente los datos estadísticos en cuanto a su acceso a la educación o al empleo. Es habitual que las mujeres con discapacidad se encuentren sobreprotegidas, infantilizadas y recluidas en el ámbito doméstico (algo que, por otro lado, las hace muy vulnerables a la violencia y las agresiones sexuales). Al mismo tiempo, esta reclusión en lo privado no les garantiza ni espacios, ni tiempos propios, ni intimidad. Viven en una tierra de nadie, no son parte de lo público ni disfrutan de las ventajas de lo privado. Además, con la discapacidad considerada fundamentalmente como problema médico, son sometidas a intervenciones innecesarias para su «normalización» y reducidas a la enfermedad (no hay persona más allá de la discapacidad). Todo esto hace que estén aisladas del mundo y, sobre todo, de otras mujeres en su misma situación, lo que reduce su capacidad de acción, de articular sus necesidades y demandas, de establecer relaciones de solidaridad, de participación social, de crear conciencia de grupo y aprovechar las ventajas (individuales y colectivas) de la acción conjunta y la pertenencia a una comunidad de iguales.

Clave entonces para solventar estos problemas y para el desarrollo de una teoría feminista de la discapacidad fue reconocer que las mujeres con discapacidad sufren una doble discriminación: por su género y por su discapacidad. Sin embargo, como hemos visto, esto no es suficiente. Estudiosas feministas de la discapacidad como las ya citadas Jenny Morris o Rosemarie Garland-Thomson van más allá. De hecho, la importancia que gran parte de la literatura sobre mujeres con discapacidad da a la doble discriminación ha sido incluso criticado. Por ejemplo, la propia Morris afirma que «centrarse en 'género y discapacidad' no debería significar la llamada 'doble discriminación' que experimentan las mujeres discapacitadas ${ }^{20}$. Morris considera que enfatizar la doble discriminación de las mujeres con discapacidad las coloca en una posición de víctimas y tiene un efecto desempoderante para ellas, a la vez que les da un carácter de añadido o de apéndice («es mujer y, además, tiene una discapacidad», lo que pone mayor énfasis en la discriminación de género

20. MORRIS, Jenny. «Feminism, gender and disability»... Op. cit., p. 4.

Feminismo/s 13, junio 2009, pp. 33-48 
y sólo de forma secundaria en la discapacidad, y viceversa, «tiene una discapacidad y, además, es mujer»). Hablar de doble discriminación, según Morris, no logra entrelazar las dos categorías y analizarlas en conjunto, sino que las separa como dos ámbitos diferenciados.

El acercamiento propuesto por Rosemarie Garland-Thomson proviene de la teoría feminista y ofrece una alternativa al de la doble discriminación: pasar del análisis sexo-género al cuestionamiento de la propia noción de «mujer».

Al complicar la identidad compartida «mujer» y reconocer que todas las mujeres tienen identidades múltiples, el feminismo académico ha generado un amplio abanico de áreas críticas de estudio -«Black Feminist Studies», «Lesbian Studies», «Latina Studies», etc.-, reconociendo cada una de ellas que ninguna mujer es simplemente una «mujer» (...) Las líneas de pensamiento feminista con mayor aplicación para los estudios de la discapacidad son aquéllas que van más allá de un enfoque exclusivo sobre el género y realizan una amplia crítica sociopolítica de las relaciones de poder sistémicas y no equitativas basada en categorías sociales asentadas en el cuerpo (...) En pocas palabras, las formas, a menudo en conflicto y siempre complejas, en que el feminismo ha politizado la materialidad de los cuerpos y ha reescrito la categoría «mujer» combinan exactamente los métodos que deberían utilizarse para analizar la discapacidad ${ }^{21}$.

El punto de vista filosófico y realizado desde las humanidades es importante, ya que la perspectiva exclusivamente sociológica ofrece una visión parcial. De hecho, el análisis filosófico explica los hallazgos sociológicos y, a su vez, se apoya en ellos. En consecuencia, la relación entre analizar la situación de las mujeres con discapacidad y tratar de reflexionar desde un punto de vista teórico sobre las ideologías que subyacen en el status quo es de reciprocidad. Es clave pensar sobre los conceptos y lo que suponen para poder interpretar de forma adecuada los datos sobre la situación de las mujeres. De este modo, será posible también diseñar acciones para mejorar las cosas; no sólo para paliar aspectos concretos y específicos, sino para actuar sobre los factores estructurales que hacen que las cosas sean como son. Por otro lado, incorporar la perspectiva de la discapacidad al feminismo o, mejor dicho, entrelazarlas, favorecerá en último término una mejor comprensión del mundo en el que vivimos, de lo que significa ser una persona, de las experiencias humanas. No sólo hay que buscar la aplicación práctica y concreta de los conocimientos, sino valorarlos en la medida en que enriquecen la reflexión sobre el hecho de que somos seres humanos y estamos en este mundo y también en cómo podemos interpretar la historia e interpretarnos a nosotras mismas.

21. Garland-Thomson, Rosemarie. Re-shaping, Re-thinking... Op. cit., pp. 3-4.

Feminismo/s 13, junio 2009, pp. 33-48 


\section{Referencias bibliográficas}

ARNAU Ripollés, M ${ }^{a}$ Soledad. «El desencuentro afortunado de dos mundos: el feminismo de la diversidad funcional (discapacidad)». II Congreso Estatal Fundación Isonomía «Mujeres Jóvenes: ¿Los nuevos feminismos?». Castellón, Universitat Jaume I, Fundación Isonomía para la Igualdad de Oportunidades, 2006.

Asch, Adrienne, Tiffany S. Perkins, Michele Fine y Harilyn Rousso. «Disabilities and Women. Deconstructing Myths and Reconstructing Realities». Encyclopedia of Women and Gender, Volume One. New York, Academic Press, 2001, pp. 345-354.

BORDO, Susan. «Normalisation and Resistance in the Era of the Image». En S. Kemp y J. Squires (eds.), Feminisms. Oxford y New York, Oxford University Press, 1997, pp. 451-455.

CARSON, Fiona. «Feminism and the Body». En S. Gamble (ed.), The Routledge Companion to Feminism and Postfeminism. London y New York, Routledge, 2001, pp. 117-128.

Gamble, Sarah (ed.). The Routledge Companion to Feminism and Postfeminism. London y New York, Routledge, 2001.

GARLAND-THOMSON, Rosemarie. Re-shaping, Re-thinking, Re-defining: Feminist Disability Studies. Washington D.C., Center for Women Policy Studies, 2001.

- «Integrating Disability, Transforming Feminist Theory». NWSA Journal. Bloomington, Fall 2002, 14 (3), pp. 1-32.

Hillyer, Barbara. Feminism and disability. Norman and London, University of Oklahoma Press, 1993.

HoGAN, Katie. Women take care. Gender, race and the culture of AIDS. Ithaca and London, Cornell University Press, 2001.

KemP, Sandra y Judith SQuires (eds.). Feminisms. Oxford y New York, Oxford University Press, 1997.

MeEKosha, Helen. «Gender and Disability (Draft entry for the forthcoming Sage Encyclopaedia of Disability)». Sydney, University of New South Wales, 2004, <http://www.leeds.ac.uk/disability-studies/archiveuk/meekosha/meekosha. pdf> consultado 2009-05-22.

Moreno Álvarez, Alejandra. Lenguajes comestibles: Anorexia, bulimia y su descodificación en la ficción de Margaret Atwood y Fay Weldon. Palma, Universitat de les Illes Balears, Treballs Feministes, 2009.

MORRIS, Jenny. «Feminism, gender and disability». Ponencia presentada en un seminario en Sidney, Australia, 1998, <http://www.leeds.ac.uk/disabilitystudies/archiveuk/morris/gender\%20and\%20disability.pdf> consultado el 22-05-2009. 
Pane, Lina. Triple Disadvantage: Women from Non-English Speaking Backgrounds Living in Australia. Canberra, The National Women's Consultative Council, 1994, <http://www.wwda.org.au/nesb.htm> consultado 2009-05-25.

- «Women - Moving Beyond The Disability». Perth, 4th International Abylimpics Conference, Women With Disabilities Australia, 1995, <http://www.wwda.org.au/moving.htm> consultado 2009-05-22.

Ríu, Carmen y Laura VIÑUELA. Módulo específico de formación en género y discapacidad. Oviedo, Fundación FASAD, 2004.

Russo, Mary. The Female Grotesque: Risk, Excess, and Modernity. New York, Routledge, 1994.

SHERWIN, Susan. No Longer Patient: Feminist Ethics and Health Care. Philadelphia, PA, Temple University Press, 1992.

SILVERS, Anita. «Feminist Perspectives on Disability». Stanford Encyclopedia of Philosophy, 2009, <http://plato.stanford.edu/entries/feminism-disability/> consultado 2009-05-22.

TRAUSTADOtTIR, Rannveig. "Obstacles to equality: the double discrimination of women with disabilities». Syracuse University, Center on Human Policy, 1990, <http://dawn.thot.net/disability.html> consultado 2009-05-22. 